\title{
Génétique épidémiologique et moléculaire de l'obésité humaine
}

\section{Epidemiological and molecular genetics in human obesity}

Oléagineux, Corps Gras, Lipides. Volume 7, Numéro 1, 86-90, Janvier - Février 2000, Dossier : actes des Journées Chevreul "Corps gras, nutrition et santé, questions d'actualité" (Bordeaux, Pessac)

Auteur(s) : Albert IRON, Laboratoire de pathologie moléculaire et de thérapie génique, Université Victor-Segalen, Bordeaux 2, 33076 Bordeaux Cedex.

\section{Author(s) : Albert IRON}

Résumé : L'obésité est une pathologie majeure pour plusieurs raisons qu'il est difficile de hiérarchiser. D'abord parce qu'elle est commune, concernant aujourd'hui près d'un individu sur trois dans les pays industrialisés. Ensuite, parce qu'elle est grave en raison des complications nombreuses et sévères, parmi lesquelles on peut citer des cardiopathies, le diabète, l'HTA, des cancers, etc. Enfin, parce que répondant à la définition de maladie multifactorielle résultant de l'interaction entre des facteurs héréditaires et des facteurs environnementaux, sa connaissance a beaucoup progressé ces dernières années, notamment grâce à l'apport de la génétique moléculaire. À l'échelon individuel et d'une manière simple, on peut définir l'obésité comme résultant d'un excès de tissu adipeux qui va se traduire par un poids élevé par rapport à ce qu'on peut considérer comme étant un poids idéal donné par la formule de Lorentz: Poids idéal $(\mathrm{kg})=$ Taille $(\mathrm{cm})-100$ - [Taille $(\mathrm{cm})-150] / 4$ (homme) ou 2,5 (femme) Sous l'angle épidémiologique, c'est-à-dire à l'échelle des populations, l'obésité sera définie à partir de critères de nature anthropologique utilisés aussi en clinique pour typer le patient obèse. On peut prendre en compte le rapport du tour de hanche sur le tour de taille (qui permet de définir une obésité de type androïde ou gynoïde selon que ce rapport est supérieur ou inférieur à 0,85 ) ou la mesure de l'adiposité évaluée par l'appréciation des plis cutanés (en particulier le pli tricipital) ou surtout l'indice de corpulence qui est le critère majeur des épidémiologistes pour l'étude de l'obésité chez l'adulte. Cet indice est la référence pour la définition de l'obésité chez l'adulte ; il est aussi appelé indice de masse corporelle (IMC) ou indice de Quetelet; il exprime le rapport du poids (en $\mathrm{kg}$ ) au carré de la taille (en m). Il a été choisi par l'OMS en 1998 pour établir la classification de l'obésité adulte [1]. Entre 18,5 et 25 , le poids du sujet est normal, souhaitable avec un risque standard. Au-delà de 25 , on définit d'abord une zone de surpoids puis trois degrés de sévérité et de complications croissantes.

Summary : Obesity is a common and major human pathology with numerous and severe complications. It is a more and more sensitive public health problem. The epidemiological approach aims at studying the pathology at the population level. Nowadays, all over the world, there are about 250 millions of obese people (with a BMI up to $30 \mathrm{~kg} / \mathrm{m2}$ ). In France, obesity concerns from 7 to 10 percent of the adult population of the two sexes. Epidemiological studies can also lead to formulate hypothesis on the pathophysiology and the genetics of obesity. Analysis of heritability, segregation and genotype-environment interactions allow to precise the genetic comtribution in the occurrence of human obesity. Over the last years, we have noted progress in the field of molecular genetics of obesity owing to the elaboration of animal models, the use of the quantitative trait loci (QTL), 
association studies, polymorphisms and candidate genes analysis. Despite the fact that there are now some genes with major effect on obesity, on the map of the human genome, the number of loci and genes related to adiposity is dramatically increasing. So, obesity remains a model of complexity in multifactorial pathologies in human beings.

Keywords : genetic epidemiology, molecular genetics, obesity, susceptibility genes.

\section{ARTICLE}

\section{Épidémiologie de l'obésité}

L'obésité est un problème de santé publique en nette progression ces 25 dernières années chez les femmes comme chez les hommes. L'obésité n'est pas une pathologie spécifique aux pays développés (tableau 2). Certes, les pays industrialisés arrivent en tête, mais ils sont " talonnés " par les pays de I'Est de l'Europe où la pathologie ne cesse de s'aggraver. En Europe, la situation diffère entre les pays du Nord (Allemagne et Grande-Bretagne plus particulièrement) et ceux du Sud (dont la France) où l'obésité est relativement plus limitée. La prévalence de l'obésité n'est pas négligeable - jusqu'à $10 \%$ - dans les pays d'Amérique latine et du Moyen-Orient, alors qu'elle ne dépasse pas $5 \%$ dans d'autres grands pays comme I'Inde et la Chine ainsi que dans de nombreux États du tiers monde. Globalement, sur l'ensemble du monde, la prévalence moyenne est de $7 \%$ et on considère que le nombre de sujets obèses serait de l'ordre de 250 millions.

En France, plusieurs études réalisées entre 1990 et 1996, incluant un très grand nombre de sujets (présentant un IMC supérieur à 30) et concernant les deux sexes, permettent de dresser un état aussi précis que possible de l'épidémiologie de l'obésité. Il en ressort :

- un pourcentage de sujets obèses global variant entre 5 et $10 \%$;

- une progression légèrement plus sensible dans le sexe féminin ;

- des disparités régionales avec un taux d'obèses plus marqué surtout dans le Nord et dans l'Est du pays.

\section{Causes de l'obésité}

Les obésités dites " génétiques " (c'est-à-dire en relation directe avec une anomalie génique) sont très rares (nous y reviendrons par la suite). À l'inverse, des raisons uniquement d'ordre psychologique, social ou culturel peuvent être à l'origine de cas d'obésité que l'on qualifie de « non génétiques ». Mais en fait, l'obésité est essentiellement une maladie multifactorielle avec prédisposition individuelle. Elle résulte de l'interaction entre l'inné et l'acquis, c'est-à-dire entre des facteurs environnementaux (comme la nutrition, l'activité physique...) et des facteurs génétiques, ces derniers ayant une influence variant en fonction de l'environnement dans lequel l'individu est placé. Nous reviendrons plus loin sur l'état actuel de la connaissance de ces gènes de prédisposition à l'obésité. 


\section{Approches méthodologiques de l'épidémiologie génétique de l'obésité}

\section{Appréciation de l'héritabilité}

C'est une quantification des ressemblances au sein des familles. On choisit un critère qui va constituer soit un trait phénotypique unique - qui sera très souvent le calcul de I'IMC mais parfois autre comme la mesure de la masse grasse -, soit une association de plusieurs traits phénotypiques. Cette héritabilité (exprimée en termes de pourcentage ou de variance) permet ainsi de définir un risque relatif standardisé par rapport à l'âge et au sexe. Ainsi a-t-on une approche quantitative de la part des gènes et de celle de l'environnement.

Les résultats des principales études centrées sur la seule mesure de l'IMC indiquent :

- d'abord, qu'il existe une nature familiale de l'obésité (par exemple qu'il y a davantage de cas d'obésité dans les familles d'obèses que dans la population générale) ;

- ensuite, que la tendance à la concentration de l'obésité dans les familles est fonction de la sévérité : ainsi, quand le cas index de la famille a une obésité massive (IMC $>40)$, le risque d'obésité est multiplié par 5 alors qu'il n'est multiplié que par 2 quand le cas index a une obésité modérée (BMI > 30) ;

- enfin que la part de l'hérédité est très variable selon les études, de quelques pour cent à $90 \%$ (ces variations s'expliquant en partie par des différences méthodologiques d'une étude à une autre).

Une étude particulièrement importante, celle des familles du Québec [2], a intégré quatre phénotypes:

- l'IMC + la quantité de graisse sous-cutanée déterminée à partir de la somme de plis adipeux sur six sites différents ;

- le pourcentage de graisse dérivé d'une pesée hydrostatique + la quantité de masse grasse ;

- la quantité de masse maigre + la distribution de la graisse sous-cutanée ;

- la quantité de graisse viscérale mesurée par tomodensitométrie. Elle a montré que la variance génétique va de $5 \%$ avec le premier phénotype à plus de $50 \%$ avec le quatrième, suggérant que la quantité de graisse intra-abdominale était fortement influencée par les gènes, indépendamment de l'adiposité totale.

\section{Études de ségrégation}

L'objectif est ici de mesurer un trait phénotypique de l'obésité pour voir, grâce à une analyse de ségrégation, s'il est influencé par la présence d'un gène à effet majeur et, si oui, d'exprimer le mode de transmission de ce gène à effet majeur.

De nombreuses études ont mis en évidence des effets géniques spécifiques à un trait phénotypique (tableau 3) [2]. La plupart de ces études centrées sur un seul trait phénotypique ont montré I'implication de gènes à effet majeur, souvent avec transmission mendélienne récessive avec des effets polygéniques relativement élevés. 
L'effet gène à effet majeur s'exerçant sur la quantité de masse grasse comme sur celle de masse viscérale s'avère cependant incompatible avec une transmission mendélienne si l'on se réfère à l'étude des familles du Québec qui a mis en évidence des effets génétiques croisés affectant simultanément les quantités de masse grasse et de masse viscérale, qui témoignent d'un pléiotropisme génétique.

\section{Études de jumeaux et d'adoptés}

Elles ont permis de mettre en évidence l'interaction génotype-environnement dont elles constituent un des principaux moyens de mesure. II est fait appel soit à des paires de jumeaux monozygotes (MZ), soit à des paires de jumeaux dizygotes (DZ), soit à des enfants adoptés.

On a pu déterminer, d'une part, le taux de concordance, c'est-à-dire le rapport du nombre de paires (de jumeaux MZ ou DZ ou d'adoptés) où les deux sont atteints sur le nombre total de paires - et ainsi calculer l'héritabilité pour un paramètre donné de l'obésité (IMC, pli cutané, rapport poids/taille...) : la variance attribuable au génotype peut aller de 50 à $90 \%$. D'autre part, il est possible de faire une comparaison intrapaire chez les jumeaux $\mathrm{MZ}$ et de calculer un coefficient de corrélation. À titre d'exemple, on peut citer les résultats de l'étude de corrélation intrapaire de jumeaux : entre les individus de génotypes différents par comparaison avec les individus de même génotype, il y a 3 fois et 7 fois plus de variations respectivement pour le gain de masse corporelle (en réponse à une suralimentation) et pour la perte de masse corporelle (consécutive à un exercice physique) $[3,4]$.

La question de l'appréciation des effets gènes et environnement dans l'obésité n'a pas lieu de se limiter aux seuls phénotypes de l'obésité. Elle se pose aussi en termes d'analyse de la covariation génétique entre l'obésité et ses comorbidités liées au risque accru de nombreuses et graves complications de l'obésité (diabète non insulinodépendant, maladies cardio et cérébrovasculaires, troubles respiratoires, arthrose, cancers, troubles comportementaux, maladies psychiatriques...), complications pour lesquelles des facteurs génétiques de prédisposition existent et peuvent interférer avec la prédisposition génétique à l'obésité. Ceci complique singulièrement les approches d'épidémiologie génétique.

\section{Stratégie d'étude des gènes candidats à l'obésité}

Grâce aux connaissances physiopathologiques, au recueil de données cliniques et au phénotypage de l'obésité (qui prend en compte des éléments aussi divers que la prise alimentaire, la dépense énergétique, le métabolisme du tissu adipeux ou le métabolisme glucidique et lipidique, on a formulé des hypothèses sur la responsabilité de gènes impliqués dans l'obésité (gènes candidats). Une démarche semblable s'appuyant sur des études animales portant sur des modèles d'obésité monogénique (chez les rongeurs essentiellement) ou sur des modèles polygéniques définis par des locus à effets quantitatifs (LEQ) vise au même objectif mais I'utilisation d'un modèle animal impose d'atteindre un gène candidat à partir de l'élection d'une région candidate.

Une fois le gène candidat choisi, la phase suivante consiste à entreprendre des études humaines soit familiales, soit cas-témoins. Grâce aux analyses de liaison ou d'association, on validera ou non 
I'hypothèse de départ. Les enjeux essentiels de cette stratégie sont une meilleure compréhension physiopathologique de l'obésité, une estimation du risque et la conception de pistes thérapeutiques.

La stratégie résumée ci-dessus a permis d'obtenir - surtout ces trois dernières années - de très nombreux résultats pas toujours faciles à interpréter en raison de l'hétérogénéité méthodologique. Aujourd'hui, la question du degré de responsabilité des facteurs génétiques de l'obésité humaine reste difficile.

\section{Carte génétique actuelle de l'obésité}

Établie par Pérusse et al. [5], elle regroupe des éléments dont la mise en évidence a été faite par des approches très variées.

\section{Gènes dont une mutation ponctuelle est associée à des cas d'obésité}

On ne comptait que deux gènes présentant une mutation ponctuelle associée à l'obésité en 1997, celui du facteur satiétogène leptine (LEP) et celui de la protéine convertase subtilisine/ kexine de type 1 (PCSK1) alors qu'ils sont sept en 1998 (tableau 4). Les mutations ponctuelles en cause sont rencontrées très majoritairement à l'état hétérozygote. Vingt-cinq cas ont été listés, concernant 10 hommes et 15 femmes d'âge allant de 2 à 78 ans : 9 impliquent le gène codant pour le récepteur de la mélanocortine MC4R, 9 présentent une anomalie de celui de la leptine ou de celui de son récepteur hypothalamique $L E P R$. Le gène PPARG codant pour le récepteur gamma des proliférateurs de peroxysomes activés est associé à quatre cas d'obésité importante.

Locus chromosomiques impliqués dans des obésités associées à des maladies génétiques à transmission mendélienne

Ces maladies rares ou très rares présentent des tableaux cliniques complexes incluant une obésité. On y distingue d'abord cinq syndromes à transmission autosomique dominante dont le plus connu est le syndrome de Prader-Willi atteignant un nouveau-né sur 10000 : une translocation ou une délétion au niveau du locus 15q11.2-q12 est responsable d'un syndrome associant, outre une obésité, un nanisme, une arriération mentale, un diabète et une insuffisance génitale. Ensuite les six syndromes à transmission autosomique récessive regroupent surtout les syndromes polymalformatifs dits de Bardet-Biedl. Enfin, parmi les cinq syndromes liés au chromosome $X$, est classé le syndrome de Wilson-Turner (Xp21.1-q22).

\section{Gènes et locus humains issus de l'étude des modèles animaux monogéniques et polygéniques}

Le tableau 5 énumère cinq modèles monogéniques d'obésité chez les rongeurs [6] présentant les anomalies suivantes : absence de transmission du signal leptinique, absence de leptine, défaut de maturation de propeptides et de prohormones par la carboxypeptidase, anomalie de la phosphodiestérase, antagonisme du récepteur de la mélanocortine MC4-R (souris agouti).

Les modèles polygéniques, ou locus à effets quantitatifs (LEQ) ne cessent de se développer. On en a dénombré 67 en 1998 dont 58 LEQ chez la souris, 7 chez le rat et 2 chez le porc. En prenant en compte des traits phénotypiques comme la prise de poids, le pourcentage d'adiposité, la matière grasse abdominale et la leptine, on a pu mettre en évidence - avec des lod-scores variables pouvant 
aller jusqu'à 8 - cent trois liaisons génétiques avec des locus du génome humain répartis sur tous les chromosomes sauf le 18 et l'Y.

\section{Gènes provenant d'études d'association avec un trait phénotypique de l'obésité}

Au total, on compte vingt-neuf gènes pour lesquels l'association est statistiquement significative : d'une part, ceux qui ont fait l'objet d'études incluant un grand nombre de cas et de témoins et, d'autre part, ceux qui ont montré une valeur de $p$ élevée. Ils figurent dans le tableau 6.

\section{Gènes et marqueurs provenant d'études de liaison avec un trait phénotypique de l'obésité}

Ils sont une cinquantaine qui présentent une liaison statistiquement significative. Le tableau 7 a retenu les gènes et marqueurs analysés lors d'études avec des effectifs importants et donnant des valeurs de $p$ très élevées.

Dès à présent, on peut affiner la problématique de l'effet " gène » dans l'obésité humaine soit en ciblant mieux sur certains locus préalablement retenus comme bons candidats, soit en « décortiquant " certaines voies métaboliques.

D'abord, le chromosome 7 (gène LEP) et le chromosome 1 (gène LEPR). L'hormone leptine, découverte en 1994, produite par les adipocytes, est un véritable facteur de satiété intervenant dans le contrôle du poids en étant libérée dans le sang en réponse au développement de la matière grasse et en générant un signal au niveau du cerveau indiquant que le corps a absorbé suffisamment de nourriture [7].

Ensuite, le chromosome 18 avec les gènes des récepteurs des mélanocortines notamment MC3R et $M C 4 R$, dont les fonctions majeures concernent le comportement alimentaire et pourraient réguler les sécrétions de leptine et de neuropeptide Y (NPY). Dans le modèle de l'obésité de la souris agouti, dite aussi " jaune ", on a montré que le produit du gène agouti - premier gène de l'obésité cloné chez la souris [8] - exerce une double action centrale et périphérique. Au niveau du noyau hypothalamique dorsal intermédiaire, agouti stimule la production de NPY via les récepteurs MC4R et $M C 5 R$, le résultat étant une hyperphagie [9]. Au niveau phériphérique, agouti pourrait déclencher un signal calcium, se fixer sur ses récepteurs du pancréas et du tissu adipeux, induire une stimulation de leptine, la synthèse des triglycérides, la lipogenèse et inhiber la lipolyse.

Le système des protéines découplantes (notamment UCP2 et UCP3, chromosome 11) constitue la $2^{\mathrm{e}}$ voie de retour des protons vers la matrice mitochondriale, voie non couplée à la synthèse d'ATP et génératrice de chaleur, donc médiatrice de la thermogenèse [10].

Enfin, au niveau de l'adipocyte, les éléments de transduction du signal impliqués dans le contrôle de la lipogenèse sont codés par des gènes candidats importants comme par exemple ceux des récepteurs de l'adrénaline, des phosphodiestérases, des périlipines, de la kinase stimulée par I'insuline (KSI), de la kinase de la précédente (KKSI) et de la lipase hormonosensible [11].

\section{CONCLUSION}

En conclusion, la carte génomique de l'obésité humaine se complexifie d'année en année. Le fait que la quasi-totalité des chromosomes et plus de 200 locus aient été répertoriés avec leurs effets plus ou moins importants renforce "I'hypermultifactorialité » de la pathologie. Pris séparément, chacun de 
ces gènes apporte une contribution souvent peu importante mais il est vraisemblable que, dans un proche avenir, des études fondées sur des associations de gènes et marqueurs candidats - choisis sur des hypothèses physiopathologiques de plus en plus robustes - ouvrent des perspectives diagnostiques et thérapeutiques de l'obésité humaine.

\section{REFERENCES}

1. WHO (1998). Obesity : preventing and managing the global epidemic. Geneva. WHO/ NUT/NCD/98.1.

2. PERUSSE L, CHAGNON YC, RICE T, RAO DC, et al. (1998). L'épidémiologie génétique et la génétique moléculaire de l'obésité : les enseignements de l'étude des familles de Québec. Med Sci, 14 : 914-24.

3. BOUCHARD C, TREMBLAY A, DESPRES JP, et al. (1990). The response to long-term overfeeding in identical twins. N Engl J Med, 322 : 1477-82.

4. BOUCHARD C, TREMBLAY A, DESPRES JP, et al. (1994). The response to exercice with constant energy intake in identical twins. Obes Res, $2:$ 400-10.

5. PÉRUSSE L, CHAGNON YC, WEISNAGEL J, et al. (1999). The human obesity gene map : the 1998 update. Obes Res, $7:$ 111-29.

6. GUERRE-MILLO M (1998). Obésité : grandeur et servitude des modèles animaux. Med Sci, 14 : 8457.

7. CLÉMENT K (1999). Leptin and the genetics of obesity. Acta Paediatr, 428 : (Suppl.) 51-7.

8. BULTMAN SJ, MICHAUD ED, WOYCHICK RP (1992). Molecular characterization of the mouse agouti locus. Cell, $71: 1-20$.

9. FAN W, BOSTON BA, KETERSON RA, et al. (1997). Role of melanocortinergic neurons in feeding and the agouti obesity syndrome. Nature, $385: 165-8$.

10. DONG DW, HE Y, KARAS M, et al. (1997). Uncoupling protein-3 is a mediator of thermogenesis regulated by thyroid hormone, beta3-adrenergic agonist, and leptin. J Biol Chem, 272 : 34129-32.

11. LANGIN D, HOLM C, LAFONTAN M (1996). Adipocyte hormono-sensitive lipase : a major regulator of lipid metabolism. Proc Nutr Soc, 55 : 93-109. 
Illustrations

\begin{tabular}{|cll}
\hline IMC $\left(\mathbf{k g} / \mathbf{m}^{2}\right)$ & Classification & Risques ass ociés \\
\hline$<18,5$ & Déficit pondéral (maigeur) & Faibles (mais risques cliniques autres) \\
$18,5-24,9$ & Poids normal, souhaitable & Moyens \\
$25-29,9$ & Surpoids ( $p$ réobésité) & Modérément élevés \\
$30-34,9$ & Excès pondéral de classe I (obésité) & Élevés \\
$35-39,9$ & Excès pondéral de classe II (obésité forte) & Trèsélevés \\
240 & Excès pondéral de classe III (obésité massive) & Extrêmement élevés
\end{tabular}

Tableau 1. Classification des sujets adultes selon l'indice de masse corporelle (OMS, 1998).

\begin{tabular}{|c|c|c|c|c|}
\hline \multirow[t]{2}{*}{ Zon e géog raphique } & \multirow{2}{*}{$\begin{array}{c}\text { Population } \\
d^{\prime} \text { âge } \geq 15 \text { ans } \\
\text { (millions) }\end{array}$} & \multirow{2}{*}{$\begin{array}{c}\text { Prévalence } \\
\text { de Yobésité } \\
\text { (\%) }\end{array}$} & \multicolumn{2}{|c|}{$\begin{array}{c}\text { Effectif approximatif } \\
\text { de sujets obèses (millions) }\end{array}$} \\
\hline & & & Fourchette & Nombre moyen \\
\hline Pays industrialisés & 640 & $15-20$ & $96-128$ & 112 \\
\hline Pays de YEst de YEurope & 330 & $20-25$ & $66-83$ & 75 \\
\hline Inde & 535 & $0,5-1$ & $3-7$ & 5 \\
\hline Chine & 825 & $0,5-1$ & $4-8$ & 6 \\
\hline Autres pays asiatiques & 430 & $1-3$ & $4-12$ & 8 \\
\hline Afrique subsaharienne & 276 & $0,5-1$ & $1-3$ & 2 \\
\hline Amérique latine et Caraïbes & 280 & $5-10$ & $14-28$ & 21 \\
\hline Mbyen-Orient & 300 & $5-10$ & $15-30$ & 22 \\
\hline Ensemble du monde & 3616 & $\approx 7$ & & $\approx \mathbf{X 0}$ \\
\hline
\end{tabular}

Tableau 2. L'obésité dans le monde. 


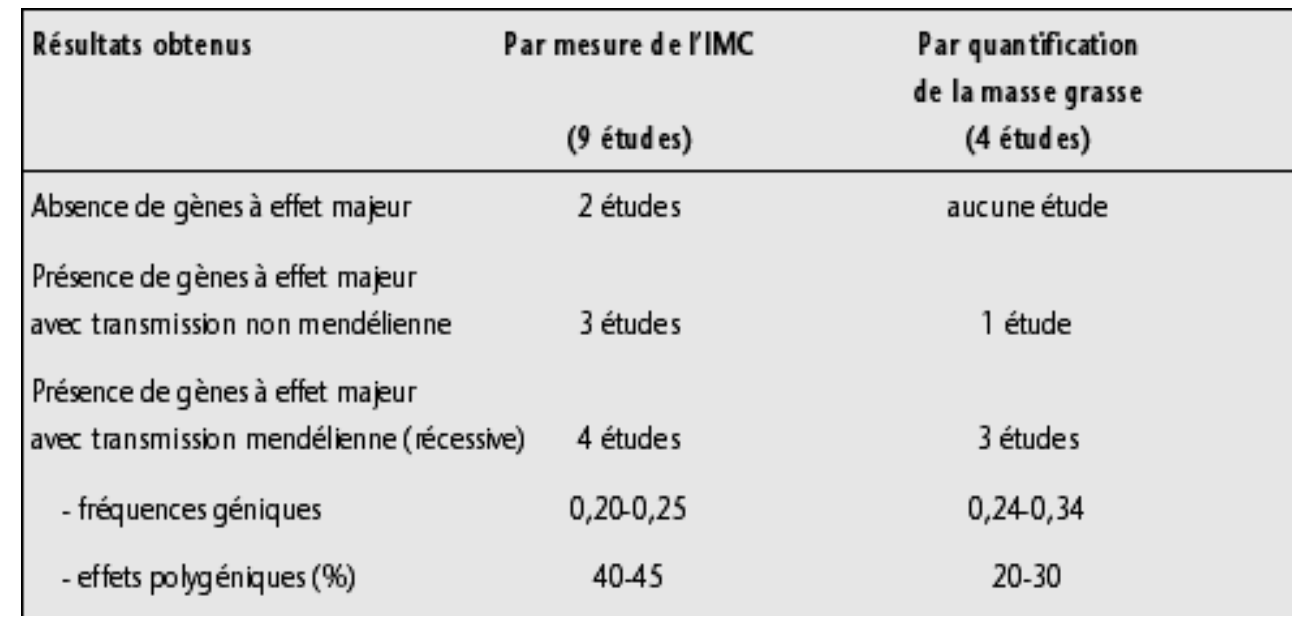

Tableau 3. Résultats cumulés des analyses de ségrégation.

\begin{tabular}{|c|c|c|c|c|c|}
\hline Gène & $\begin{array}{l}\text { Localis ation } \\
\text { chrom osom iq ue }\end{array}$ & Fon ction & $\begin{array}{l}\text { Nombre et type } \\
\text { de mutation }\end{array}$ & $\begin{array}{l}\text { Nombre } \\
\text { de cas }\end{array}$ & $\begin{array}{c}\text { Caractéristique } \\
\text { de lobésité }\end{array}$ \\
\hline LEPR & $1 p 31$ & $\begin{array}{l}\text { récepteur hormonal } \\
\text { hypotha lamique }\end{array}$ & 1 (h) & 3 & sévère \\
\hline \multirow[t]{2}{*}{ POMC } & $2 \mathrm{p} 23$ & hormone hypothalam ique & 1 (hc) & 1 & sévère et précoce \\
\hline & & proopiomélanocortine & $1(H)$ & 1 & sévère et précoce \\
\hline THRS & $3 p 24.1-p 22$ & $\begin{array}{l}\text { récepteur de Yhormone } \\
\text { thyröidienne }\end{array}$ & $1(h)$ & 1 & modérée \\
\hline PPARG & $3 p 25$ & $\begin{array}{l}\text { récepteur des proliférateurs } \\
\text { des peroxysomes activés }\end{array}$ & $1(h)$ & 4 & im portante \\
\hline PCSKI & $5 q 15-q 21$ & enzyme & $1(h)$ & 1 & - \\
\hline \multirow[t]{2}{*}{ LEP } & $7 q 31$ & hormone périphérique & 1 (h) & 2 & - \\
\hline & & & $1(h)$ & 3 & - \\
\hline \multirow[t]{2}{*}{$M C 4 R$} & $18 q 21.3$ & récepteur hormonal & $1(h)$ & 2 & - \\
\hline & & & 1 (h) & 7 & - \\
\hline
\end{tabular}

Tableau 4. Cas d'obésité associés à des gènes mutés ponctuellement. $(\mathrm{H}$ : mutation à l'état homozygote, $\mathrm{h}:$ mutation à l'état hétérozygote ; hc : hétérozygotie composite.) 


\begin{tabular}{|c|c|c|c|}
\hline Espèce & Modèle & Produit du gène & $\begin{array}{c}\text { Chrom osome humain } \\
\text { hom ologue }\end{array}$ \\
\hline Souris & dia bétique $(d b / d b)$ & récepteur de b leptine & $1 \mathrm{p} 31$ \\
\hline Souris & obèse (ob/ob) & leptine & $7 q 31$ \\
\hline Rat & $\operatorname{gras}(f a / f a)$ & carboxypeptidase & $4 q 21$ \\
\hline Souris & tubby (tub) & phosphodiestérase & 11 p1 5.1 \\
\hline Souris & yelbw $(A y)$ & agouti & $20 q 12-13.11$ \\
\hline
\end{tabular}

Tableau 5. Modèles monogéniques d'obésité chez les rongeurs.

\begin{tabular}{|c|c|c|}
\hline Gène & $\begin{array}{l}\text { Localis ation } \\
\text { chrom osom iq ue }\end{array}$ & Trait phénotypique associé \\
\hline $\begin{array}{l}\text { PPARC (récepteurs gamma des pro liférateurs } \\
\text { de peraxysomes activés) }\end{array}$ & $3 \mathrm{p} 25$ & taux de leptine \\
\hline ADRS2 (récepteu adénergique bêta 2) & $5 q 31-q 32$ & IMC, \% ma se grasse \\
\hline TNF(tumor necrosis factor) & $6 \mathrm{p} 21.3$ & IMC \\
\hline LEP (leptine) & $7 q 31.3$ & perte de poids \\
\hline ADRB3 (récepteur ad'énergique bêta 3) & 8p12-p11.3 & $\begin{array}{l}\text { IMC, tour de taille/tour de hanche, } \\
\text { obésité précoce }\end{array}$ \\
\hline UCP2 (protéine découplante 2) & $11 q 13$ & variations métaboliques sur 24 h \\
\hline MCSR (récepteu 5 de la mélano cortine) & $18 \mathrm{p} 11.2$ & IMC chez a femme \\
\hline MC4 R (récepteu 4 de la mélanocortine) & $18 \mathrm{p} 21.3$ & \% ma sse grasse chez la femme \\
\hline LIPE (lipase hormonosensible) & $19 q 13.1-q 13.2$ & obésité et diabète non insulinodépendant \\
\hline
\end{tabular}

Tableau 6. Gènes candidats pour l'obésité chez l'homme. Sélection des principales études d'association présentant une forte signification statistique. 


\begin{tabular}{|c|c|c|c|}
\hline $\begin{array}{l}\text { Localis ation } \\
\text { chrom osom ique }\end{array}$ & Gèn e ou locus & Nom & Trait phénotypique lié \\
\hline $1 p 31-p 21$ & LEPR & récepteur de b leptine & quotient respiratoire \\
\hline $1 q 21-q 22$ & D15498 & & lipodystrophie fam iliale \\
\hline $1 q 22-q 25$ & ATP1B1 & ATPase $\mathrm{Na} / \mathrm{K}$ dépenda nte 1 bêta 1 & quotient respiratoire \\
\hline $2 \mathrm{p} 12$ & D251788 & & leptine \\
\hline $6 \mathrm{p} 21.3$ & TNF & tumor necrosis factor & $\%$ de tissu adipeux \\
\hline $7 q 31$ & LEP & leptine & IMC \\
\hline $7 q 33$ & KEL & groupes sang uins Kell & IMC \\
\hline $11 q 13$ & $U C P 2$ et $U C P 3$ & protéines découplantes 2 et 3 & métabolisme au repos \\
\hline $11 q 213-q 24$ & $?$ & & $\begin{array}{l}\text { dépense énergétique } \\
\text { des } 24 \mathrm{~h}\end{array}$ \\
\hline $18 q 21$ & $?$ & & \% de tissu gras corpore \\
\hline $20 \mathrm{q} 11.2$ & $?$ & & quotient respiratoire \\
\hline
\end{tabular}

Tableau 7. Gènes (et marqueurs génétiques) candidats pour l'obésité chez l'homme. Sélection des principales études de liaison présentant une forte signification statistique. 